\title{
Efficient Computation of RCS from Scatterers of Uncertain Shapes
}

\author{
C. Chauvière \\ Laboratoire de Mathématiques, Université Blaise Pascal, 63177 Aubière Cedex, France. \\ J. S. Hesthaven \\ Division of Applied Mathematics, Brown University, Providence, Rhode Island 02912. \\ L. C. Wilcox \\ ICES, University of Texas, Austin, Texas 78712.
}

\begin{abstract}
We propose a way of accounting for the lack of detailed knowledge about material shapes in computational time-domain electromagnetics. We use Legendre-Gauss-Lobatto, Stroud-2 and Stroud-3 quadrature formulae to solve the resulting stochastic equation and we show the efficiency of the proposed method over statistical Monte-Carlo simulations. We also show how the radar-cross-section in scattering is affected by the uncertainty in shape of the objects and by the direction of the incident field.
\end{abstract}

Index Terms - Maxwell's equations, spectral methods, uncertainty quantification.

\section{INTRODUCTION}

Most of the research effort in CEM has been in developing efficient numerical algorithms for different applications, assuming ideal inputs, boundary conditions or computational domains. While computational methods have become increasingly accurate, their reliance on exact data (e.g. the geometry of the objects, the material parameters, the sources terms, etc.) is becoming a bottleneck in the modeling of complex problems. A standard way to deal with this lack of knowledge or uncertainty, is to assume that some of the parameters are random and compute macroscopic quantities (e.g. means and variances) through Monte-Carlo sampling. In that case, one runs a deterministic code many times and computes the statistics of interest from an ensemble of solutions. The advantage of this approach is its simplicity, however the rate of convergence of Monte-Carlo is quite slow since it is proportional to $\sqrt{M}$, where $M$ is the number of samples. Therefore, designing more efficient numerical methods for the solution of stochastic partial differential equations with random inputs or random coefficients is meeting growing interest.

When the randomness can be modelled by a relatively small number of independent random variables (i.e. up to three or four), a stochastic collocation method based on Lagrange polynomials was shown to be as simple as a Monte-Carlo simulation, but with higher rates of convergence [2][13]. In the present paper, the uncertainty is modelled by a number of independent random variables in $O(10)$, which precludes from using this approach. Instead, we use a method based on Stroud-2 and Stroud-3 quadrature rules, for the computation of multiple integrals. To compute single integrals we will use the
Legendre-Gauss-Lobatto quadrature rule. Here again, it will be shown to possess the simplicity of Monte-Carlo simulations but with higher convergence rates.

This paper is organized as follows: in Section 2, we recall the deterministic Maxwell's equations in the time-domain and we give some details of its spatial discretization using a highorder discontinuous Galerkin method. In Section 3, we give some details about the computation of the RCS. In Section 4, we describe the modelling of a general object having a random shape, and we explain how our problem can be reduced to solving Maxwell's equations on a fixed mesh but with random coefficients. The second part of Section 4 is devoted to the discretization of the random space based on Legendre-GaussLobatto, Stroud-2 and Stroud-3 quadrature rules. This sets the stage for numerous examples in Section 5. In Section 6 and 7, we conclude and offer some suggestions for continued research in this direction.

\section{MAXWELL'S EQUATIONS AND ITS NUMERICAL APPROXIMATION}

The time-dependent Maxwell's equations in the scattered field formulation are given as

$$
\begin{aligned}
\epsilon \frac{\partial \mathbf{E}^{s}}{\partial t} & =\nabla \times \mathbf{H}^{s}+\sigma \mathbf{E}^{s}+\mathbf{S}^{E} \\
\mu \frac{\partial \mathbf{H}^{s}}{\partial t} & =-\nabla \times \mathbf{E}^{s}+\mathbf{S}^{H}
\end{aligned}
$$

where, $\mathbf{E}^{s}$ and $\mathbf{H}^{s}$ denote the scattered electric and magnetic fields, $\varepsilon(\mathbf{x})$ and $\mu(\mathbf{x})$ are the local permittivity and permeability, $\sigma(\mathbf{x})$ is the conductivity of the media and $\mathbf{S}^{E}$ and $\mathbf{S}^{H}$ are source terms. Here we have not explicitly written the divergence constraints assuming that the initial conditions satisfy these constraints. Taking the divergence of equations (1)(2) verifies that if the initial conditions satisfy the divergence constraints then the solution to Maxwell's equations (1)-(2) will also satisfy the divergence constraints.

Let the incident field $\left(\mathbf{E}^{i}, \mathbf{H}^{i}\right)$ be a solution to Maxwell's equations in a media of permittivity, permeability, and conductivity $-\varepsilon^{i}(\mathbf{x}), \mu^{i}(\mathbf{x}), \sigma^{i}(\mathbf{x})$, respectively. Along a perfect electric conductor (PEC), the boundary conditions on the total electric field $\mathbf{E}^{t}=\mathbf{E}^{i}+\mathbf{E}^{s}$ and the total magnetic field 


$$
\mathbf{H}^{t}=\mathbf{H}^{i}+\mathbf{H}^{s} \text { are }
$$

$$
\begin{aligned}
\hat{\mathbf{n}} \times \mathbf{E}^{t} & =\mathbf{0} \\
\mathbf{H}^{t} \cdot \hat{\mathbf{n}} & =0,
\end{aligned}
$$

where $\hat{\mathbf{n}}$ is the outward pointing normal vector at the surface.

We now briefly describe the computational methods used for solving Maxwell's equations (1)-(2) in the physical space. A discontinuous Galerkin method is used; which offers a number of advantages over widely used alternative (see [6], [7], [8], [9], for example) and we shall simply sketch its main components. First, we rewrite Maxwell's equations (1)-(2) in conservation form

$$
\mathbf{Q} \frac{\partial \mathbf{q}}{\partial t}+\nabla \cdot \mathbf{F}(\mathbf{q})=\mathbf{S}
$$

where $\mathbf{q}$ is the state vector given by

$$
\mathbf{q}=\left(\begin{array}{c}
\mathbf{E} \\
\mathbf{H}
\end{array}\right)
$$

and the components of the tensor $\mathbf{F}$ are defined by

$$
\mathbf{F}_{i}(\mathbf{q})=\left(\begin{array}{c}
-\mathbf{e}_{i} \times \mathbf{H} \\
\mathbf{e}_{i} \times \mathbf{E}
\end{array}\right),
$$

where $\mathbf{e}_{i}$ denotes the Cartesian unit vectors. On the righthand side of (5), $\mathbf{S}=\left[\mathbf{S}^{E}, \mathbf{S}^{H}\right]$ is the source term, which depends on the incident field, and the material matrix $\mathbf{Q}$ is a diagonal matrix with values $(\epsilon, \epsilon, \epsilon, \mu, \mu, \mu)$ on its diagonal. We assume that the computational domain, $\Omega$, is tessellated by triangles in two spatial dimensions and tetrahedrons in three spatial dimensions. Given an element $D$ of the tessellation, the represent the local solution $\mathbf{q}_{N}$ restricted to $D$ is given as

$$
\mathbf{q}_{N}(\mathbf{x}, t)=\sum_{i=1}^{N} \widetilde{\mathbf{q}}_{i}(t) L_{i}(\mathbf{x}),
$$

where $\widetilde{\mathbf{q}}_{i}$ reflects nodal values, defined on the element. The function $L_{i}(\mathbf{x})$ signifies an $p$ th order Lagrange polynomial $(N=(p+1)(p+2) / 2$ for triangles and $N=(p+1)(p+$ $2)(p+3) / 6$ for tetrahedrons), associated with grid points on the reference element (see [6], [7], [8], [9] for details). The discrete solution, $\mathbf{q}_{N}$, of Maxwell's equations is required to satisfy

$$
\begin{aligned}
& \int_{D}\left(\mathbf{Q} \frac{\partial \mathbf{q}_{N}}{\partial t}+\nabla \cdot \mathbf{F}\left(\mathbf{q}_{N}\right)-\mathbf{S}_{N}\right) L_{i}(\mathbf{x}) d \mathbf{x} \\
= & \oint_{\partial D} \hat{\mathbf{n}} \cdot\left[\mathbf{F}\left(\mathbf{q}_{N}\right)-\mathbf{F}^{*}\right] L_{i}(\mathbf{x}) d \mathbf{x} .
\end{aligned}
$$

In (9), $\mathbf{F}^{*}$ denotes a numerical flux, whose expression can be found in [6], and $\hat{\mathbf{n}}$ is an outward pointing unit vector defined at the boundary $\partial D$ of the element $D$. Note that this is an entirely local formulation, and relaxing the continuity of the elements decouples the elements, resulting in a blockdiagonal global mass matrix which can be trivially inverted in preprocessing. After discretization of the operators and evaluation of the integrals appearing in (9), the problem can be rewritten in matrix-vector form (see [7])

$$
\mathbf{Q M} \frac{d \mathbf{q}_{N}}{d t}+\mathbf{S} \cdot \mathbf{F}_{N}-M \mathbf{S}_{N}=\mathbf{F} \hat{\mathbf{n}} \cdot\left[\mathbf{F}_{N}-\mathbf{F}^{*}\right]
$$

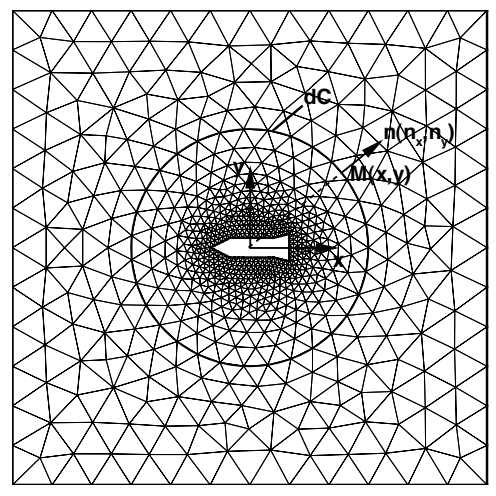

Fig. 1. Illustration showing the scattering object and a possible contour $d C$ to compute the RCS.

The matrices $\mathbf{M}, \mathbf{S}$, and $\mathbf{F}$ represent the local mass-, stiffness-, and face-integration matrices, respectively, the exact entries of which only depend on the metric of the triangle (see [6]). The local nature of the scheme allows for the use of an explicit solver for the time discretization of (10) and this is done using an explicit fourth-order Runge-Kutta method.

\section{Computation of the Radar Cross Section (RCS)}

Given the importance of the RCS in this paper, we devote this section to explaining how it is computed. For the sake of simplicity, we will give details for the 2D transverse magnetic case. We assume that the scattered fields $E_{z}(x, y, t)$, $H_{x}(x, y, t)$ and $H_{y}(x, y, t)$ are available at any point $(x, y)$ of the computational domain and at any time $t$. When a somewhat established regime is reached, we assume that the electric field can be written under the form

$$
E_{z}(x, y, t)=E_{z}^{0}(x, y) \cos \left(2 \pi f t+\phi_{E_{z}}\right) .
$$

The two unknowns that need to be determined in the above expression are $E_{z}^{0}(x, y)$ and $\phi_{E_{z}}$. They are computed by taking two solutions $E_{z}\left(x, y, t_{1}\right)$ and $E_{z}\left(x, y, t_{2}\right)$ at time $t_{1}$ and $t_{2}$ and by solving the system

$$
\left\{\begin{array}{l}
E_{z}\left(x, y, t_{1}\right)=E_{z}^{0}(x, y) \cos \left(2 \pi f t_{1}+\phi_{E_{z}}\right) \\
E_{z}\left(x, y, t_{2}\right)=E_{z}^{0}(x, y) \cos \left(2 \pi f t_{2}+\phi_{E_{z}}\right)
\end{array} .\right.
$$

Then, we define the phasor of the electric field $\widetilde{E}_{z}$ to be the complex-valued field defined at each point $(x, y)$ by

$$
\widetilde{E}_{z}(x, y)=E_{z}^{0}(x, y) e^{j \phi_{E z}}
$$

The same procedure can be applied to the magnetic field, leading to the phasors

$$
\widetilde{H}_{x}(x, y)=H_{x}^{0}(x, y) e^{j \phi_{H_{x}}}
$$

and

$$
\widetilde{H}_{y}(x, y)=H_{y}^{0}(x, y) e^{j \phi_{H_{y}}} .
$$


We now take any contour $d C$ that surrounds the scattering object (see Figure 1). Although the RCS is independent of the chosen contour $d C$, the computation of the RCS will be greatly simplified by taking a contour that matches the finite element mesh. To compute the RCS all we need is the phasor of the electric field, the phasor of the magnetic field and the normal unit vector on the contour $d C$. The RCS under the scattering angle $\phi$, is a function of the following integral along $d C$ :

$$
\begin{aligned}
F(\phi)= & \frac{1}{2} e^{j \frac{\pi}{4}} \oint_{d C}\left[\left(n_{y} \widetilde{H}_{x}-n_{x} \widetilde{H}_{y}\right)+\right. \\
& \left.\left(n_{x} \cos \phi+n_{y} \sin \phi\right) \widetilde{E}_{z}\right] \\
& e^{j 2 \pi f(x \cos \phi+y \sin \phi)} d l
\end{aligned}
$$

Since we have chosen a contour $d C$ that matches the finite element mesh, it is formed by (say) $K$ non-overlapping segment lines $\left[a_{i}, b_{i}\right](i=1, \ldots, K)$ such that $d C=\bigcup_{i=1}^{K}\left[a_{i}, b_{i}\right]$. Therefore the integral (16) becomes

$$
\begin{aligned}
F(\phi)= & \frac{1}{2} e^{j \frac{\pi}{4}} \sum_{i=1}^{K} \int_{a_{i}}^{b_{i}}\left[\left(n_{y} \widetilde{H}_{x}-n_{x} \widetilde{H}_{y}\right)+\right. \\
& \left.\left(n_{x} \cos \phi+n_{y} \sin \phi\right) \widetilde{E}_{z}\right] \\
& e^{j 2 \pi f(x \cos \phi+y \sin \phi)} d l,
\end{aligned}
$$

or, after a simple change of variable

$$
\begin{aligned}
F(\phi)= & \frac{1}{2} e^{j \frac{\pi}{4}} \sum_{i=1}^{K}\left(\frac{b_{i}-a_{i}}{2}\right) \int_{-1}^{1}\left[\left(n_{y} \widetilde{H}_{x}-n_{x} \widetilde{H}_{y}\right)+\right. \\
& \left.\left(n_{x} \cos \phi+n_{y} \sin \phi\right) \widetilde{E}_{z}\right] \\
& e^{j 2 \pi f(x(\eta) \cos \phi+y(\eta) \sin \phi)} d \eta .
\end{aligned}
$$

Then, the integral on the interval $[-1,1]$ is computed using a Legendre-Gauss-Lobatto quadrature rule to give

$$
\begin{aligned}
F(\phi)= & \frac{1}{2} e^{j \frac{\pi}{4}} \sum_{i=1}^{K}\left(\frac{b_{i}-a_{i}}{2}\right) \sum_{l=1}^{p+1} w_{l} \\
& {\left[\left(n_{y}^{l} \widetilde{H}_{x}^{l}-n_{x}^{l} \widetilde{H}_{y}^{l}\right)+\left(n_{x}^{l} \cos \phi+n_{y}^{l} \sin \phi\right) \widetilde{E}_{z}^{l}\right] } \\
& e^{j 2 \pi f\left(x^{l} \cos \phi+y^{l} \sin \phi\right)},
\end{aligned}
$$

where $w_{l}$ are the quadrature weights and $(p+1)$ is the number of collocation points on each sides of the triangles. Note that the phasors $\widetilde{E}_{z}^{l}, \widetilde{H}_{x}^{l}$ and $\widetilde{H}_{y}^{l}$ do not need to be interpolated since each one is readily available at all collocations points (in particular, at collocations points on the sides of the triangles). Finally, the RCS under the scattering angle $\phi$ is given by

$$
R C S(\phi)=2 \pi f \frac{|F(\phi)|^{2}}{\left|\mathbf{E}_{0}\right|^{2}},
$$

where $\mathbf{E}_{0}$ is defined in equation (32). For the $2 D$ calculations, no scaling is applied to the RCS, i.e. the plots of the RCS on a $d B$ scale are simply

$$
R C S_{d B}(\phi)=10 \log _{10}(R C S(\phi)) .
$$

For the plots of the RCS for $3 D$ scattering objects, a scaling equal to $\lambda^{2}$ is applied.

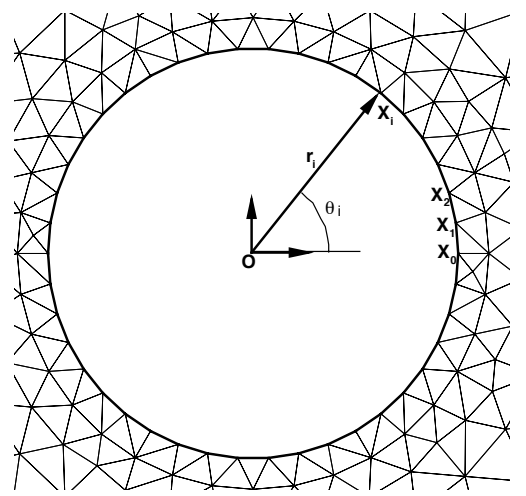

Fig. 2. Points $X_{i}=\left(r_{i} \cos \left(\theta_{i}\right), r_{i} \sin \left(\theta_{i}\right)\right)$ defining the boundary of an object.

\section{ACCOUNTING FOR UNCERTAINTY}

\section{A. Modelling of a random surface}

We consider an object whose shape can vary in a random fashion. As an example, Figure 2 shows a disc which is modelled by $(n-1)$ line segments $\left[\mathbf{X}_{i} \mathbf{X}_{i+1}\right]_{1 \leq i \leq n-1}$ from a finite element mesh. Those line segments are defined by the points of polar coordinates $\mathbf{X}_{i}=\left(r_{i} \cos \left(\theta_{i}\right), r_{i} \sin \left(\theta_{i}\right)\right)_{1 \leq i \leq n}$. We now assume that the point $\mathbf{X}_{i}$ can be moved randomly by a quantity $\delta \mathbf{X}_{i}=\left(\delta r_{i} \cos \left(\theta_{i}\right), \delta r_{i} \sin \left(\theta_{i}\right)\right)_{1 \leq i \leq n}$ to take a new position $\left(\mathbf{X}_{i}+\delta \mathbf{X}_{i}\right)=\left(\left(r_{i}+\delta r_{i}\right) \cos \left(\theta_{i}\right),\left(r_{i}+\delta r_{i}\right) \sin \left(\theta_{i}\right)\right)_{1 \leq i \leq n}$. We further assume that two points (say $\mathbf{X}_{i}$ and $\mathbf{X}_{j}$ ) with polar angles $\theta_{i}$ and $\theta_{j}$ close to each other should have a random height $\left\|\delta \mathbf{X}_{i}\right\|$ close to $\left\|\delta \mathbf{X}_{j}\right\|$. This is done by introducing the covariance matrix $\mathbf{K}$ such that:

$$
K_{i j}=c^{2} e^{-\frac{\left|\beta_{i}-\beta_{j}\right|}{b}},
$$

where

$$
\beta_{i}=\left\{\begin{array}{ccc}
\theta_{i} & \text { if } & \theta_{i} \in[0, \pi] \\
\pi-\theta_{i} & \text { if } & \left.\left.\theta_{i} \in\right] 0,2 \pi\right]
\end{array}\right.
$$

In the definition of $\beta_{i}$ we have to separate the cases $\theta_{i} \in$ $[0, \pi]$ and $\left.\left.\theta_{i} \in\right] 0,2 \pi\right]$ to ensure that two points on the random surface, one with a polar angle close to 0 and the other one with a polar angle close to $2 \pi$, be strongly correlated. In the relation (20), $b$ is a parameter which can control how correlated two points $\mathbf{X}_{i}$ and $\mathbf{X}_{j}$ can be and $c$ is another parameter which controls the roughness of the surface (the magnitude of $\left\|\delta \mathbf{X}_{i}\right\|$ for $i=1, . ., n$ is directly proportional to $c$ ). Furthermore, $c$ is the standard deviation of each component of $\delta \mathbf{r}$.

To generate a random surface, the problem can be formulated as follows: find a random vector $\delta \mathbf{r}=$ $\left(\delta r_{1}, \delta r_{2}, \ldots, \delta r_{n}\right)^{T}$ with a given covariance matrix $\mathbf{K}$ that will generate the new random surface $\left(\mathbf{X}_{i}+\delta \mathbf{X}_{i}\right)=\left(\left(r_{i}+\right.\right.$ $\left.\left.\delta r_{i}\right) \cos \left(\theta_{i}\right),\left(r_{i}+\delta r_{i}\right) \sin \left(\theta_{i}\right)\right)_{1 \leq i \leq n}$. This procedure is illustrated in Figure 3 , where a triangle $\left(A, \mathbf{X}_{i}, \mathbf{X}_{i+1}\right)$ having one 


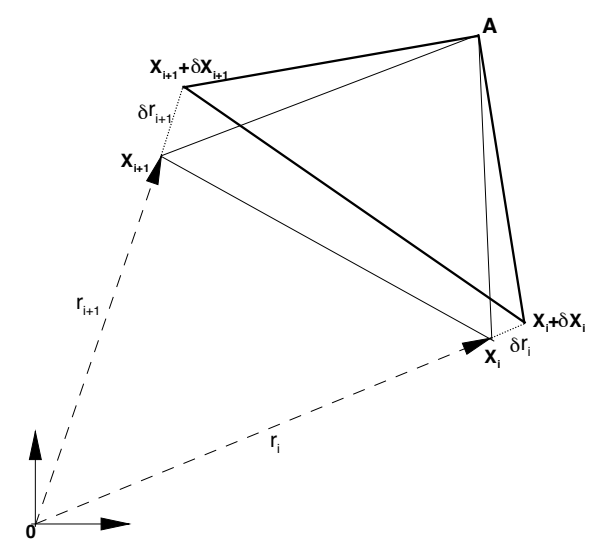

Fig. 3. Illustration showing how the points on the boundary of an object are moved randomly.

side $\left[\mathbf{X}_{i} \mathbf{X}_{i+1}\right]$ sitting on the boundary of the object is distorted into a new triangle $\left(A, \mathbf{X}_{i}+\delta \mathbf{X}_{i}, \mathbf{X}_{i+1}+\delta \mathbf{X}_{i+1}\right)$ which side $\left[\left(\mathbf{X}_{i}+\delta \mathbf{X}_{i}\right)\left(\mathbf{X}_{i+1}+\delta \mathbf{X}_{i+1}\right)\right]$ will compose the new shape of the object. From a practical point of view, this can be easily done by first generating a vector $\mathbf{G}=\sqrt{3}\left(\alpha_{1}, \alpha_{2}, \ldots, \alpha_{n}\right)^{T}$, where $\alpha_{1}, \alpha_{2}, \ldots, \alpha_{n}$ are random independent variables following (for example) a uniform law on the interval $[-1,1]$. Since $\mathbf{K}$ is symmetric definite positive, it can be decomposed as $\mathbf{K}=\mathbf{P D} \mathbf{P}^{-1}$, where $\mathbf{D}$ is a diagonal matrix with positive eigenvalues on its diagonal. Then, it can be shown that the vector $\delta \mathbf{r}$ given by $\delta \mathbf{r}=\mathbf{P D}^{1 / 2} \mathbf{G}$ will be a random vector with covariance matrix $\mathbf{K}$. In terms of implementation, one generates independents pseudo-random numbers $\alpha_{1}, \alpha_{2}, \ldots, \alpha_{n}$ with a uniform law on the interval $[-1,1]$, then the vector $\delta \mathbf{r}$ is given by the matrix-vector product $\mathbf{P} \mathbf{D}^{1 / 2} \mathbf{G}$ (the matrix $\mathbf{P D}^{1 / 2}$ can be pre-computed and stored once for all). The coordinates of the points of the new random surface will be $\left(\mathbf{X}_{i}+\delta \mathbf{X}_{i}\right)$ for $i=1, \ldots, n$. The procedure described here for a disc can be easily adapted to objects with more general shapes, as will be shown in the numerical experiments section.

As an example, we have considered a somewhat simplified rocket in Figure 4. Figure 5 shows a zoom of its front part for the original (non-distorted) rocket and a typical sample mesh, when the procedure described above has been applied (here, we take $b=5$ and $c^{2}=0.002$ in equation (20)). It should be noted that with this procedure, one just needs to generate a single mesh for the problem to be solved (the mesh of the object with its original shape, i.e. the mesh of Figure 4, for example). One needs to be careful that once the points defining the original object have been moved randomly, the triangles sitting on the object do not distort the mesh too much. This can be easily controlled by the parameter $c$ in equation (20) which is directly linked to the amplitude that $\delta r_{i}$ can take. The coordinates of the points which define the finite element mesh only occur in the matrices $\mathbf{M}, \mathbf{S}$, and $\mathbf{F}$ of equation (10) and those quantities are constant element by element. Since those matrices appear as multiplicative coefficients into Maxwell's

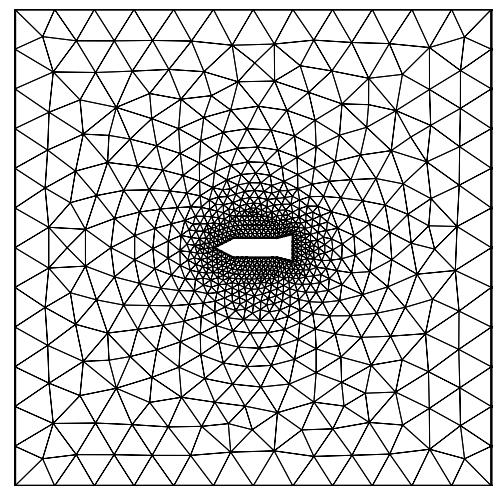

Fig. 4. Original mesh for the rocket problem.

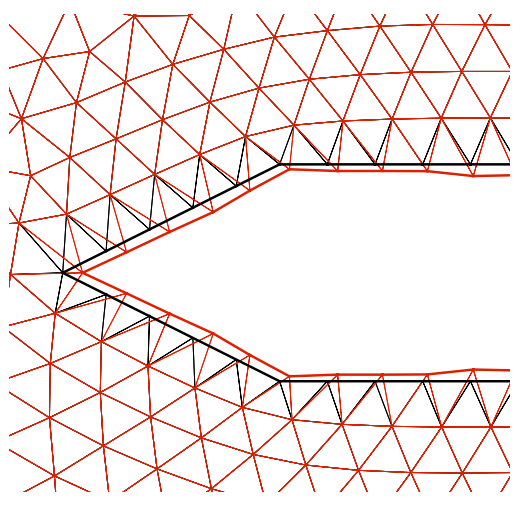

Fig. 5. One sample of a mesh for the rocket with a random shape together with its original shape.

equations we have transformed a problem with an object having a random shape (which usually requires the generation of a new mesh for each sample) into an equivalent stochastic problem having a fixed mesh but with random coefficients.

\section{B. Monte-Carlo simulation}

A Monte-Carlo simulation is therefore quite simple: one simply need to generate (say) $M$ random vectors $\left\{\delta \mathbf{r}_{m}\right\}_{1 \leq m \leq M}$ as described above. Each set of random numbers will give a new random surface from which we can compute $M$ solutions of Maxwell's equations. Those $M$ solutions of Maxwell's equations, will give $M$ radar cross section $\left\{R C S_{m}\right\}_{1 \leq m \leq M}$ from which we can compute averages as follows:

$$
<R C S>=\frac{1}{M} \sum_{m=1}^{M} R C S_{m}
$$


And for the variance, we have

$$
\left.\operatorname{var}(R C S)=\frac{1}{M} \sum_{m=1}^{M}\left(R C S_{m}\right)^{2}-<R C S\right\rangle^{2} .
$$

It should be noted that the advantage of the Monte-Carlo approach is its simplicity (it only requires repetitive runs of an existing deterministic solver), but it is hard to get accurate solutions due to its slow rate of convergence $O\left(M^{-1 / 2}\right)$. In the next subsection, we will introduce a stochastic collocation method which has the simplicity of the Monte-Carlo approach but with better rates of convergence.

\section{Stochastic collocation method based on Stroud's points}

About fifty years ago, Stroud [11] constructed a set of cubature points to compute integrals of the form

$$
I[f]=\int_{[-1,1]^{n}} f(\mathbf{x}) d \mathbf{x} .
$$

This set of cubature points based on $(n+1)$ points is exact for polynomials of degree two, and the approximation is written as

$$
I[f] \simeq \sum_{i=1}^{n+1} \omega_{i} f\left(\mathbf{x}_{i}\right)
$$

where the $(n+1)$ cubature points $\mathbf{x}_{i}=\left(x_{i}^{1}, x_{i}^{2}, \ldots, x_{i}^{n}\right)$ are given by

$$
\begin{aligned}
x_{i}^{2 r-1} & =\sqrt{\frac{2}{3}} \cos \left(\frac{2 r(i-1) \pi}{n+1}\right), \\
x_{i}^{2 r} & =\sqrt{\frac{2}{3}} \sin \left(\frac{2 r(i-1) \pi}{n+1}\right),
\end{aligned}
$$

for $r=1, \ldots,[n / 2]$, and if $n$ is odd, $x_{i}^{n}=(-1)^{(i-1)} / \sqrt{3}$. The weights in (25) are all equal to $2^{n} /(n+1)$. Similarly, we have the Stroud-3 method based on $2 n$ points which is exact for polynomials of degree three :

$$
I[f] \simeq \sum_{i=1}^{2 n} \omega_{i} f\left(\mathbf{x}_{i}\right)
$$

where the $2 n$ cubature points $\mathbf{x}_{i}=\left(x_{i}^{1}, x_{i}^{2}, \ldots, x_{i}^{n}\right)$ are now defined by

$$
\begin{aligned}
x_{i}^{2 r-1} & =\sqrt{\frac{2}{3}} \cos \left(\frac{(2 r-1) i \pi}{n}\right), \\
x_{i}^{2 r} & =\sqrt{\frac{2}{3}} \sin \left(\frac{(2 r-1) i \pi}{n}\right),
\end{aligned}
$$

for $r=1, \ldots,[n / 2]$ and if $n$ is odd, similarly we have, $x_{i}^{n}=(-1)^{i} / \sqrt{3}$. The weights in (27) are all equal to $2^{n} / 2 n$. It can be shown [10] that Stroud-2 and Stroud-3 methods employ the minimal number of points for their corresponding integration accuracy. Since Stroud-2 and Stroud-3 methods appeared, many other cubature formulae have been established to compute various high-dimensional integrals. In the 70's, Stroud published a book [12] containing most cubature formulae known at that time. This extensive work was then continued by Cools in a series of two papers [3][4]. The idea of the stochastic collocation method is based on polynomial interpolations in the multi-dimensional random space. We assume that Lagrange polynomials based on Stroud's cubature points exist, and we express the RCS using the Lagrange interpolation polynomials, which gives (for Stroud-2 cubature points)

$$
R C S(\mathbf{x})=\sum_{i=1}^{n+1} R C S_{i} L_{i}(\mathbf{x})
$$

where $\left\{L_{i}\right\}_{1 \leq i \leq n+1}$ are $(n+1)$-variate Lagrange polynomials based on points $\left\{\mathbf{x}_{i}\right\}_{1 \leq i \leq n+1}$ of the cubature formula (25). We note that by construction of Lagrange polynomials, we have $L_{i}\left(\mathbf{x}_{j}\right)=\delta_{i j}$ and therefore $R C S_{i}=R C S\left(\mathbf{x}_{i}\right)$. The radar cross sections $R C S\left(\mathbf{x}_{i}\right)$ can be easily computed as follows:

- for each cubature point $\mathbf{x}_{i}$ generate a new surface with $\delta \mathbf{r}_{i}=\mathbf{P} \mathbf{D}^{1 / 2} \mathbf{G}_{i}$ and $\mathbf{G}_{i}=\sqrt{3} \mathbf{x}_{i}(i=1, \ldots, n+1)$

- compute the corresponding solution of Maxwell's equations

- compute the corresponding radar cross section $R C S\left(\mathbf{x}_{i}\right)$

The expression of the RCS is now available under the form (29) and we will show that statistical quantities, e.g. mean and variance, can easily be computed. By taking the average of equation (29) and evaluating the multi-dimensional integral with Stroud-2 cubature formula (25), we get

$$
\begin{aligned}
& <R C S(\mathbf{x})>=\frac{1}{2^{n}} \sum_{i=1}^{n+1} R C S_{i} \int_{[-1,1]^{n}} L_{i}(\mathbf{x}) d \mathbf{x} \\
& =\frac{1}{2^{n}} \sum_{i=1}^{n+1} R C S_{i} \sum_{j=1}^{n+1} \omega_{j} L_{i}\left(\mathbf{x}_{j}\right) \\
& =\frac{1}{2^{n}} \sum_{i=1}^{n+1} R C S_{i} \sum_{j=1}^{n+1} \omega_{j} \delta_{i j} \\
& =\frac{1}{2^{n}} \sum_{i=1}^{n+1} \omega_{i} R C S_{i}
\end{aligned}
$$

Similarly, for the variance, we have

$$
\operatorname{var}(R C S(\mathbf{x}))=\frac{1}{2^{n}} \sum_{i=1}^{n+1} \omega_{i} R C S_{i}^{2}-<R C S(\mathbf{x})>^{2}
$$

The same procedure can be used with Stroud-3 cubature, and in that case, $2 n$ realizations of the RCS corresponding to the $2 n$ cubature points of equation (27) will have to be computed. When the random space is one-dimensional this procedure can also be repeated for the Legendre-Gauss-Lobatto quadrature.

Alternatively, we could have used orthogonal polynomials to express the RCS, i.e. equation (29) would have to be replaced by

$$
R C S(\mathbf{x})=\sum_{i=1}^{n+1} R C S_{i} \Phi_{i}(\mathbf{x})
$$


where $\Phi_{i}(\mathbf{x})$ are orthogonal polynomial on $[-1,1]^{n}$. The expression (31) is usually referred as "polynomial chaos expansion" and was used in a number of mechanical problems by Ghanem [5]. The problem of this approach lies in the difficulty to compute in an efficient way the coefficients $R C S_{i}$.

\section{Reduced random space}

For the stochastic collocation method, we have seen that there is a close relation between the size of the random space $n$ and the number of calls to the deterministic Maxwell solver (i.e. we need $(n+1)$ calls for Stroud- 2 and $2 n$ calls for Stroud3). A way of reducing the CPU cost of the method would be to reduce the size of the random space $n$. This is possible, up to a certain extent, depending on the covariance matrix $\mathbf{K}$ defined by equation (20). Let us consider the two extreme cases:

First, we assume that $b \rightarrow 0$ and $c=1$ in equation (20); in this case, $\mathbf{K}=\mathbf{I}$ is the identity matrix and $\mathbf{P}=\mathbf{D}=\mathbf{I}$. In other words we will need $n$ independent and identically distributed random variables to describe the process since all points of the object are uncorrelated.

Now we assume that $b \rightarrow \infty$ and $c=1$ in equation (20); in this case, all variables are fully correlated. We have $K_{i j}=P_{i j}=1$ for $i, j=1, \ldots, n ; D_{11}=1$ and $D_{i i}=0$ for $i=2, \ldots, n$. Thus, all $n$ points of the sample $\delta \mathbf{r}=\left(\delta r_{1}, \delta r_{2}, \ldots, \delta r_{n}\right)^{T}=\mathbf{P D}^{1 / 2} \mathbf{G}$ are moved with the same amplitude, i.e. $\delta r_{1}=\delta r_{2}=\ldots=\delta r_{n}$. This means that $n=1$ would suffice to describe the problem. In practice, we are between those two extreme cases and the size of the random space can be given by the number of significant eigenvalues of $\mathbf{D}$ denoted by $m$ (with $m \leq n$ ). Then, the modified algorithm proceeds as follows:

- Compute the $n$ eigenvalues $\left\{\lambda_{i}\right\}_{1 \leq i \leq n}$ of the matrix $\mathbf{K}$ and select the $m$ most significant ones satisfying $\left|\frac{\lambda_{i}}{\lambda_{\max }}\right| \geq T O L$, where $T O L$ is some small number. The integer $m$ will be the size of the random space needed to move the points of the objects randomly.

- We denote by $\widetilde{\mathbf{P}}$ and $\widetilde{\mathbf{D}}$ the matrices of size $n \times m$ constructed from the restriction of the matrices $\mathbf{P}$ and $\mathbf{D}$ where we have only kept the most significant eigenvalues and their associated eigenvectors.

- Since the dimension of the random space is reduced to $m \leq n$, the collocation points $\mathbf{x}$ are $m$-dimensional vectors based on quadrature points for the computation of $m$-dimensional integrals. For Stroud-2, we have $(m+1)$ points and for Stroud-3, it is $2 m$.

- The vector $\delta \mathbf{r}$ used to move the mesh is now given by $\delta \mathbf{r}=\left(\delta r_{1}, \delta r_{2}, \ldots, \delta r_{n}\right)^{T}=\widetilde{\mathbf{P}} \widetilde{\mathbf{D}}^{1 / 2} \mathbf{G}$, where $\mathbf{G}=\sqrt{3} \mathbf{x}$.

In the next section, we will study three different problems with three different kind of meshes: a cylinder (simple smooth mesh), a square (simple non-smooth mesh) and a rocket (general non-smooth mesh). Table I gives typical values of $m$ for those three problems for different values of the parameter $b$ of equation (20). The threshold value for the most significant eigenvalues was taken equal to TOL $=10^{-3}$ (ratio of the smallest eigenvalues to the largest ones). We can see that the size reduction of the random space can increase from a factor of two (when $b=1$ ) up to a factor of approximately five (when $b=10$ ).

\begin{tabular}{|l|l|l|l|}
\hline$b$ & 1 & 5 & 10 \\
\hline cylinder problem $(n=42)$ & 21 & 16 & 9 \\
\hline square problem $(n=44)$ & 22 & 16 & 9 \\
\hline rocket problem $(n=45)$ & 22 & 14 & 10 \\
\hline
\end{tabular}

TABLE I

SIZE OF THE REDUCED RANDOM SPACE $m$ FOR THREE PROBLEMS AND THREE DIFFERENT VALUES OF THE PARAMETER $b$ OF EQUATION (20).

\section{NUMERICAL EXPERIMENTS}

The ideas described above are tested on three different problems in two spatial dimensions and two different problems in three spatial dimensions. We consider a transverse magnetic plane wave scattering by an object of boundary $\Gamma$ and in all the two-dimensional cases considered here, the source term $\mathbf{S}^{E}$ of equation (1) is due to a transverse magnetic plane wave and takes the form :

$$
\mathbf{S}^{E}=\left\{\begin{array}{ccc}
\mathbf{E}_{0} \sin (2 \pi f(\mathbf{k} \cdot \mathbf{x}-t)) & \text { if } \quad \begin{array}{c}
\mathbf{x} \in \Gamma \\
\mathbf{0}
\end{array}
\end{array},\right.
$$

where the wave vector $\mathbf{k}$ is taken equal to $\mathbf{k}=(-1,0)$ and the frequency $f$ is taken equal to 1 (or 3 for the high frequency cases). For all cases with two spatial dimensions a PML is used to truncate the domain (see [1] for details) and the solution in the physical space is obtained with degree four (or six when $f=3$ ) Lagrange polynomials in each spectral element (see equation (8)). For the cases with three spatial dimensions a sponge layer is used for the absorbing boundary condition to truncate the domain.

\section{A. Smooth shape}

We first consider a 2D circular cylinder with a radius of $\lambda$. Here $\lambda$ ie the wavelength of the incident plane wave. The mesh consists of 852 elements (see Figure 6) and the dimension of the full random space is 42 , i.e. the cylinder is formed by 42 points of the finite element mesh. The parameters $b$ and $c^{2}$ in equation (20) are taken equal to 1 and 0.001 , respectively. This choice of $c$ corresponds to the radial randomness having a standard deviation of about $0.032 \lambda$. According to table I, it is possible to reduce the size of the random space to $m=21$. In order to justify the choice of $T O L=10^{-3}$ as a threshold value for the most significant eigenvalues, we have represented the variance of the RCS for three different values $\left(T O L=10^{-1}\right.$, $T O L=10^{-2}$ and $T O L=10^{-3}$ ) as well as the variance of the RCS for the full random space when Stroud-3 method is used (see Figure 7). We see that for $T O L=10^{-1}$ (in that case, the size of the random space is reduced to $m=4$ ), the variance of the RCS is far from its expected value. When $T O L=10^{-2}$ (which corresponds to $m=14$ ), the variance of the RCS gets close to the one with a full random space and when $T O L=10^{-3}$, they are indistinguishable. For the average of the RCS the four curves match exactly and therefore they are not shown. 


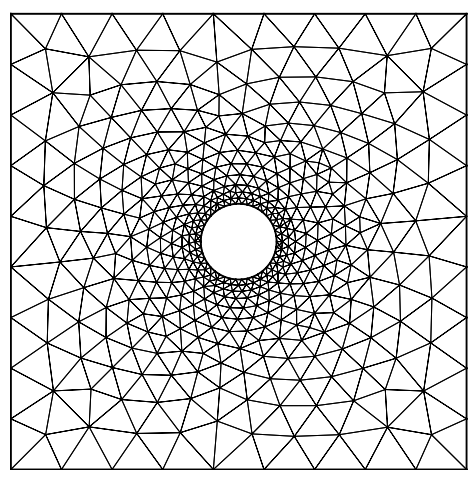

Fig. 6. Original mesh for the cylinder problem.

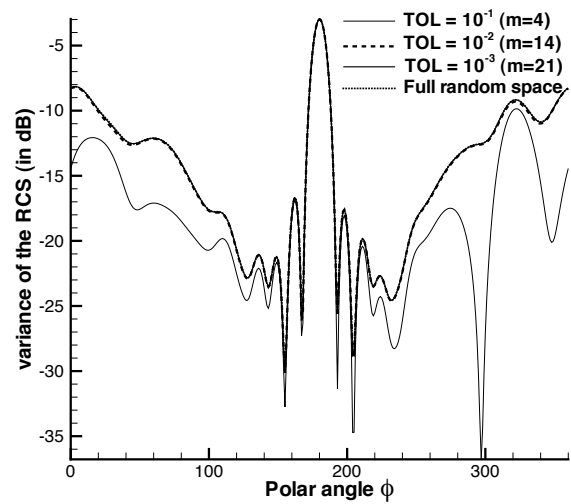

Fig. 7. Comparison of the variance of the RCS for reduced random spaces and for the full random space.

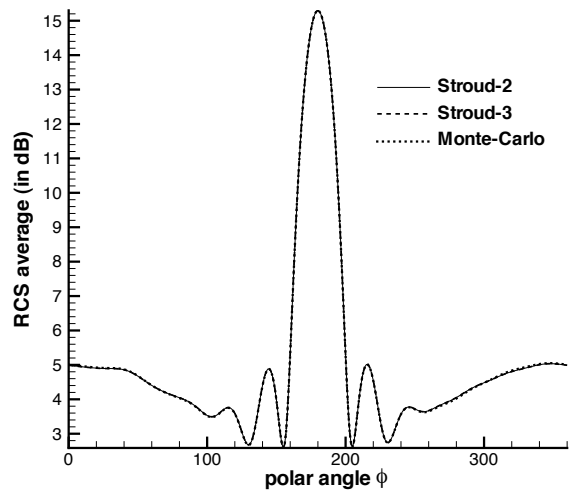

Fig. 8. Comparison of the average of the RCS for Stroud-2, Stroud-3 and Monte-Carlo methods.

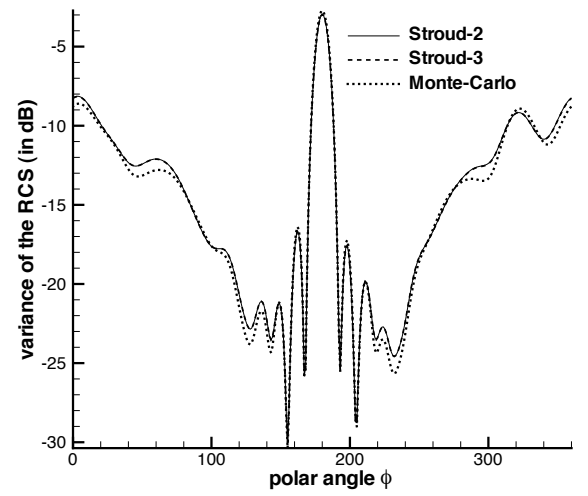

Fig. 9. Comparison of the variance of the RCS for the cylinder problem using Stroud-2, Stroud-3 and Monte-Carlo methods.

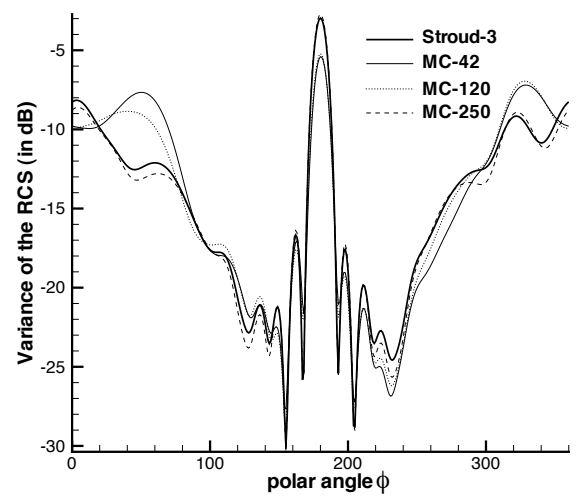

Fig. 10. Comparison of the variance of the RCS for the cylinder problem using Stroud-3 method and Monte-Carlo method with 42, 120 and 250 samples.

Figures 8 and 9 show the average and the variance of the RCS for the Stroud-2, the Stroud-3 and the Monte-Carlo method using 250 samples. We see that for the average, all methods have converged. For the variance, the Stroud-2 and Stroud-3 have converged to the same RCS but the Monte-Carlo method give slightly different results. Figure 10 shows that as we increase the number of samples of the Monte-Carlo method from 42 to 250 , the variance of the RCS converges to the solution of the Stroud-3 method. Therefore, it can be expected that a Monte-Carlo simulation with more samples would give results closer to the Stroud results. Note that the CPU cost of the Monte-Carlo method with 42 samples is exactly the same as the one of Stroud-3 method and when 250 samples are used, it is about six times more CPU expensive than Stroud-3 (the cost is proportional to the number of samples solutions computed, i.e. for Stroud-2 it is $m+1=22$; for Stroud-3, it is 


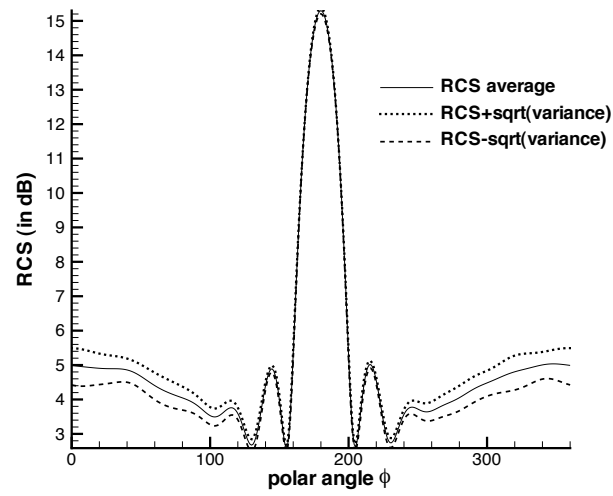

Fig. 11. RCS for the cylinder problem. Results are shown with the mean RCS as well as \pm one standard deviation (shown results are for Stroud-3).

$2 m=42$ and for the most accurate Monte-Carlo simulation, it is 250 samples). Finally, Figure 11 shows the average of the RCS and the possible variations around its average value when Stroud-3 method is used. It can be noted that for this test case the uncertainty in shape affects the RCS mostly in the sidebands.

\section{B. Non-smooth shape}

We now consider a square of width $0.8 \lambda$ whose mesh consists of 840 elements. Again, $\lambda$ is the wavelength of the incident plane wave. The dimension of the full random space is 44 , which means that the square is formed by 44 points of the finite element mesh. The parameters $b$ and $c^{2}$ in equation (20) are taken equal to 5 and 0.001 , respectively. This choice of $c$ corresponds to the radial randomness having a standard deviation of about $0.032 \lambda$. By choosing $b=5$, the covariance matrix $\mathbf{K}$ defined by (20) is more diagonal dominant than it was in the previous example, and the number of most significant eigenvalues is reduced to $m=16$. Due to the geometrical singularities in the corners of the square, this is a much harder problem to solve than the cylinder problem. This is illustrated on Figure 12 and 13, where we show the mean and the variance of the RCS for the Stroud-2, the Stroud-3 and the Monte-Carlo methods using 250 samples. We can see on Figure 12 that the mean converges to the same values for the three methods. However, for the variance, the Stroud-3 and the Monte-Carlo methods give similar results but the Stroud2 method gives results which have not converged, especially in the sidebands. This is because Stroud-2 can only integrate exactly multi-variate polynomials of degree two at most, and the exact solution of this example cannot be accurately represented by such polynomials. On the other hand, Stroud3 which can integrate exactly multi-variate polynomials of degree three at most does a better job. In order to emphasize the cost saving of Stroud-3 method over Monte-Carlo method, we have represented the variance of the RCS for Stroud-3, Monte-Carlo with 32 samples (which has the same CPU-cost

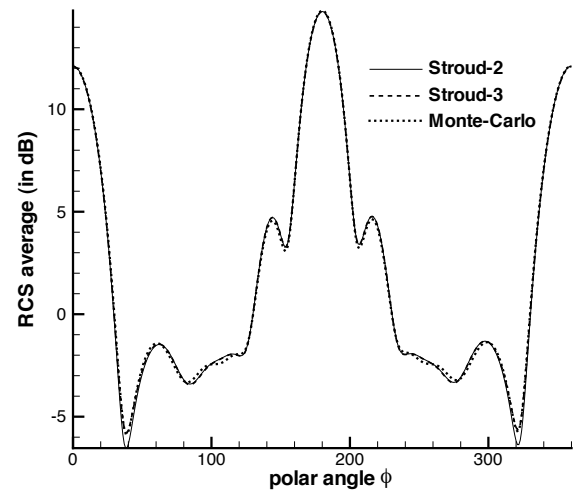

Fig. 12. Comparison of the average of the RCS for the square problem for Stroud-2, Stroud-3 and Monte-Carlo methods.

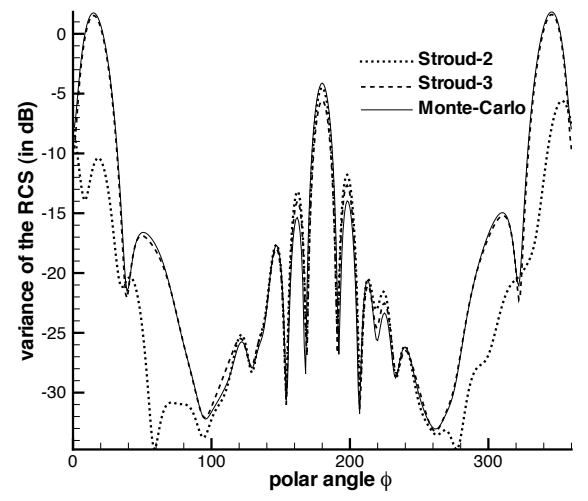

Fig. 13. Comparison of the variance of the RCS for the square problem using Stroud-2, Stroud-3 and Monte-Carlo methods.

as the Stroud-3 method) and Monte-Carlo with 250 samples in Figure 14. We see that as we increase the number of samples, the Monte-Carlo solution converges slowly to the Stroud-3 solution. Figure 15 shows the average of the RCS and the possible variations around its average value obtained with Stroud-3 cubature. We can see that like for the cylinder problem, the uncertainty in shape affects the RCS mostly in the sidebands.

\section{General shape}

As a last shape, we consider the (simple) rocket shown in Figure 4 which is $\lambda$ long and the body is $0.25 \lambda$ wide. The mesh consists of 1465 elements and the dimension of the full random space is 45 (i.e. the rocket is formed by 45 points of the finite element mesh). As for the square problem, the parameters $b$ and $c^{2}$ in equation (20) are taken equal to 5 and 0.001 , respectively and the size of the random space 


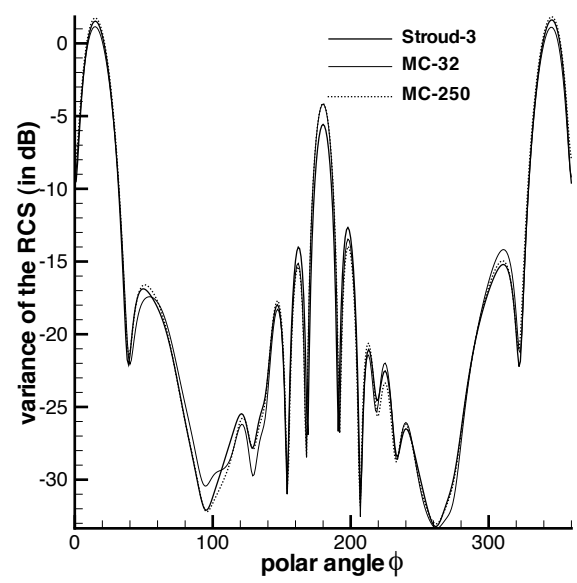

Fig. 14. Comparison of the variance of the RCS for the square problem using Stroud-3 method and Monte-Carlo method with 32 and 250 samples.

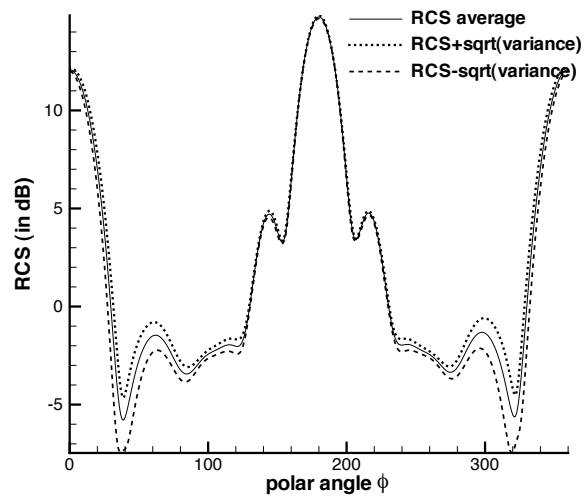

Fig. 15. RCS for the square problem. Results are shown with the mean RCS as well as \pm one standard deviation.

can be reduced to $m=14$. This choice of $c$ corresponds to the radial randomness having a standard deviation of about $0.032 \lambda$. Similarly to the square problem, good convergence of the average RCS is obtained both for Stroud-2, Stroud-3 and Monte-Carlo simulations. However for the variance of the RCS, only Stroud-3 and Monte-Carlo with enough samples (i.e. more than 250) give good results. Figure 16 shows the average of the RCS and the possible variations around its average value obtained with Stroud-3 cubature. For this case, the uncertainty in shape affects the RCS both in the middle part and in the sidebands.

\section{Higher frequency cases}

As a last numerical experiment in two spatial dimensions, we increase the frequency $f$ of the source term (32) up to $f=$ 3 . This causes the radius of the cylinder to increase to $3 \lambda$ and the size of the rocket to increase to $3 \lambda$ long and $0.75 \lambda$ wide in

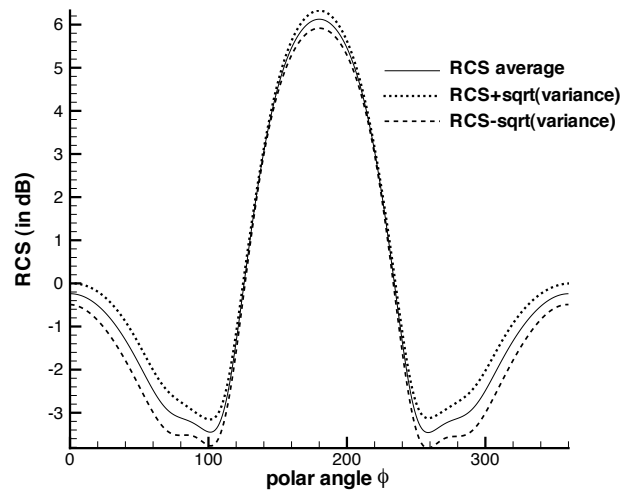

Fig. 16. RCS for the rocket problem. Results are shown with the mean RCS as well as \pm one standard deviation.

the body. For these cases the radial randomness has a standard deviation of about $0.095 \lambda$. For all the previous numerical experiments, we have used 4th order elements in each triangle of the finite element grid. Due to the higher frequency, all the results shown in this section are obtained with 6th order elements to get converged results in the physical space (i.e. the value of $p$ defined below equation (8) is increased to $p=6$ ). Figures 17 and 18 show the average of the RCS and the possible variations around its average value obtained with Stroud-3 cubature for the cylinder and the rocket problem, respectively. The form of the uncertainty remains the same as in the previous numerical examples, the only difference being the frequency of the source term. By comparing Figure 11 with Figure 17 and Figure 16 with Figure 18, we see that as we increase the frequency, the regions where the RCS has the greatest variance remains more or less the same. In this numerical experiment, convergence of the average of the RCS and the variance of the RCS is good for the cylinder problem, for both Stroud and Monte-Carlo methods. However, for the rocket problem only Stroud-3 and Monte-Carlo with enough samples give converged results for the variance.

\section{E. Sphere}

For the first experiment with three spatial dimensions we consider the scattering of a transverse magnetic plane wave from a PEC sphere. We assume the sphere has a uniform random radius in the interval $[0.9 \lambda, 1.1 \lambda]$, where $\lambda$ is the wavelength of the incident field. The use of one random variable is not a limitation of the method but is done for logistical reasons to have reasonable computation times. Since this experiment has only one random dimension, a sixth order Legendre-Gauss-Lobatto quadrature is used for collocation in the random space. For the spatial discretization we use fourth order elements and a sample mesh is presented in Figure 19 which is restricted to the surface of the sphere. Figure 20 shows the average of the RCS and the possible variations 


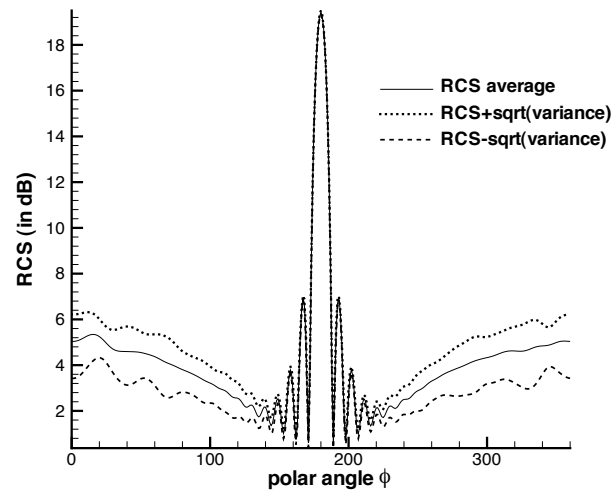

Fig. 17. RCS for the cylinder problem at higher frequency. Results are shown with the mean RCS as well as \pm one standard deviation.

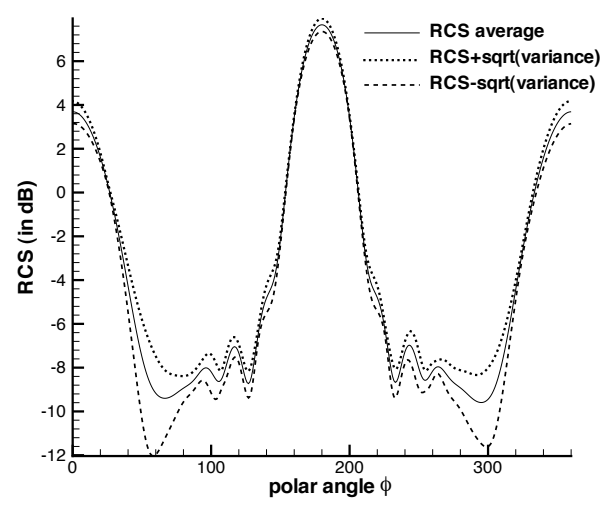

Fig. 18. RCS for the rocket problem at higher frequency. Results are shown with the mean RCS as well as \pm one standard deviation.

around its average value. The uncertainty in the radius of the sphere affects the RCS mainly in the sideband.

\section{F. Three-dimensional rocket}

For the second experiment with three spatial dimensions we consider the scattering of a transverse magnetic plane wave from a PEC rocket. The orientation of the scattering plane and the scattering angle $\theta$ is is given in Figure 21 along with a geometric description of the rocket. The scattering angle of the incident field is assumed to be random with uniform distribution in the interval $\left[10^{\circ}, 20^{\circ}\right]$. As in the case for the sphere, the use of one random variable is not a limitation of the method but is done for logistical reasons to have reasonable computation times. A fourth Legendre-Gauss-Lobatto is used for collocation in the one-dimensional random space. For this calculation the physical space is discretized with degree five polynomials in each element. Figure 22 shows the mesh used

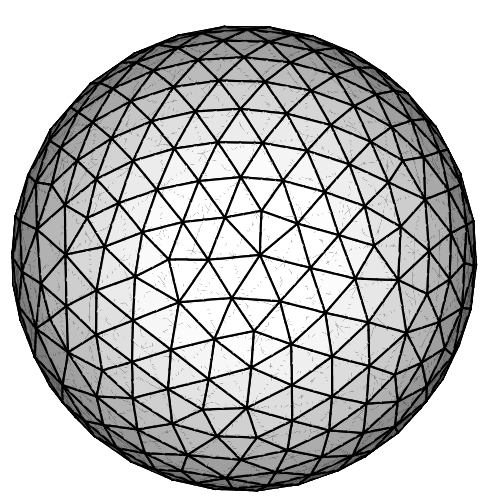

Fig. 19. One sample of a surface mesh for the sphere with a random radius.

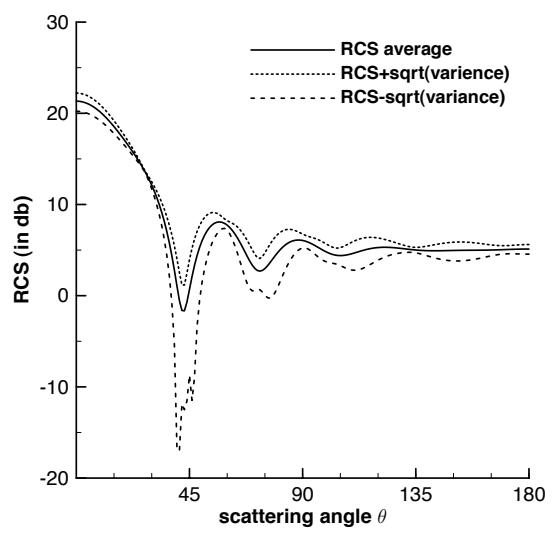

Fig. 20. RCS for the sphere problem. Results are shown with the mean RCS as well as \pm one standard deviation.

restricted to the surface of the rocket and Figure 23 shows the average of the RCS and the possible variations around its average value. The uncertainty in the direction of the incident field affects the RCS mainly near the local maxima and minima points of the RCS.

\section{Possible EXTENSIONS TO OTHER TYPES OF UNCERTAINTIES}

For convenience the numerical examples presented in three spacial dimensions only use one random dimension unlike the two-dimension examples which used multi-dimensional random spaces. This restriction to one random dimension was done only to keep the total computational time tractable. The method presented for applying the randomness in two spacial dimension should generalize to three dimensions with an appropriate choice of the correlation between neighboring points in three dimensions.

So far, we only have discussed PEC objects with random shapes and uncertainties in the incident field, however the approach described above can equally be used for other types of uncertainties. For example, instead of being purely 


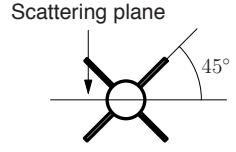

End View

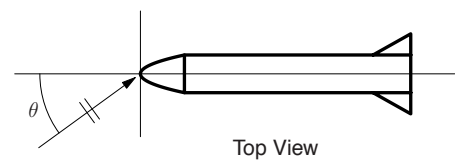

(a)

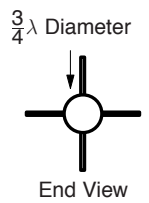

End View

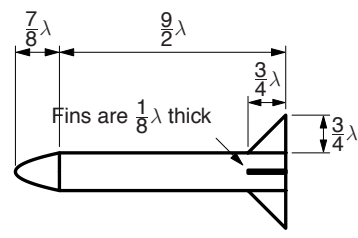

(b)
Side View
Fig. 21. The orientation of the rocket with respect to the scattering plane is given in (a). Here $\theta$ is the scattering angle. The top view presents a projection of the rocket onto the scattering plane. The geometric description of the threedimensional rocket is given in (b). Here $\lambda$ is the wavelength of the incident field.

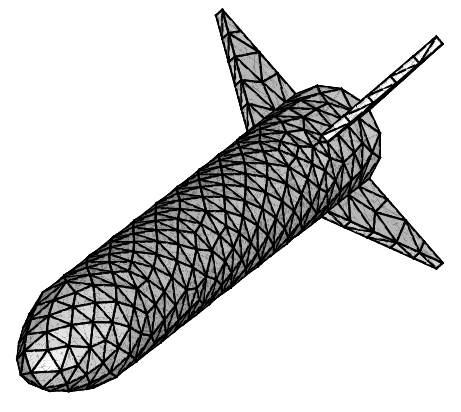

Fig. 22. The surface mesh for the three-dimensional rocket.

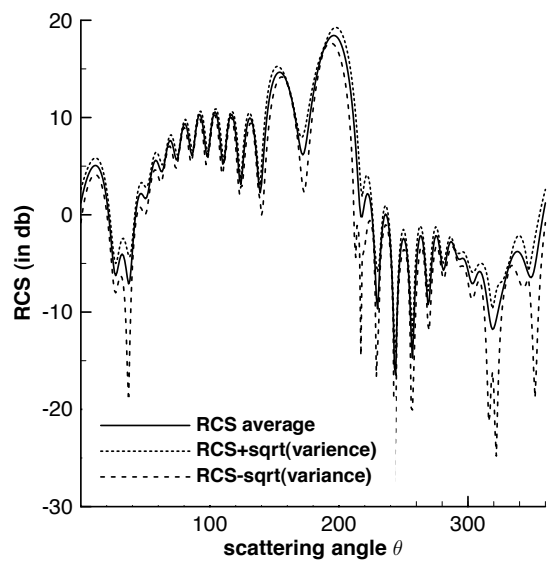

Fig. 23. RCS for the three-dimension rocket problem. Results are shown with the mean RCS as well as \pm one standard deviation. reflective, the object can be material with a random shape. In that case, it is necessary to mesh the entire domain and define a permittivity $\varepsilon$ that will takes some value inside the object and another value outside. For material objects, the shape of the objects can be moved randomly in the same way as a PEC object. In [2], the uncertainty in shape of a material object was studied and the approach used was limiting the uncertainty to be modeled by a single random variable. Other types of uncertainties were also studied (randomness of the source term to mimic a slight variation in the frequency of the source, randomness of the permittivity), however the method used was only efficient for random spaces of small dimension. The approach presented in this paper is more general since it allows efficient simulations, even when the size of the random space is large.

\section{CONCLUSION}

In this paper, we have proposed a way to model the uncertainty in shape for different objects and study its influence on the RCS, computed from the solution of the time-domain Maxwell's equations. It was shown that the modeling of the object with a random shape could be rewritten into a problem with a fixed shape but with Maxwell's equations having random coefficients. We have proposed an efficient way of solving this stochastic equation based on Stroud's cubature formulae. The proposed approach has a number of significant advantages: it is as simple as a Monte-Carlo simulation and for a given level of accuracy, it requires less outcomes to be computed. We have shown that for non-smooth objects (like the square), it was preferable to use the Stroud-3 method instead of the Stroud-2 one. For all the examples with two spatial dimensions treated here, the number of independent random variables used to generate the random surface was between 14 and 21 , showing the efficiency of the proposed method for a relatively high dimension of the random space. We have also presented results for simulations in three spatial dimensions with a single random variable.

\section{ACKNOWLEDGEMENT}

The last two authors appreciate the partial support of AFOSR under grant FA9550-04-1-0072.

\section{REFERENCES}

[1] S. Abarbanel, D. Gottlieb, and J. S. Hesthaven, Long time behavior of the perfectly matched layer equations in computational electromagnetics, J. Sci. Comput., 17 (2002), pp. 405-422.

[2] C. Chauvière, J. S. Hesthaven and L. Lurati, Computational modeling of uncertainty in time-domain electromagnetics, SIAM J. Sci. Comput., 28 (2006), pp. 751-775

[3] R. Cools and P. Rabinowitz, Monomial cubature rules since 'Stroud': A compilation, J. Comput. Appl. Math. 48 (1993), pp. 309-326

[4] R. Cools, Monomial cubature rules since 'Stroud': A compilation -part 2, J. Comput. Appl. Math. 112 (1999), pp. 21-27

[5] R. Ghanem and P. Spanos, Stochastic Finite Elements: a Spectral Approach, Springer-Verlag (1991).

[6] J. S. Hesthaven and T. Warburton, High-order nodal methods on unstructured grids. I. Time-domain solution of Maxwell's equations, J. Comput. Phys., 181 (2002), pp. 1-34.

[7] J. S. Hesthaven and T. Warburton, High-order accurate methods for timedomain electromagnetics, CMES-Computer Modeling in Engineering and Sciences, 5 (2004), pp. 395-408. 
[8] J. S. Hesthaven and T. Warburton, High-order nodal discontinuous Galerkin methods for the Maxwell eigenvalue problem, Philos. Trans. R. Soc. Lond. Ser. A Math. Phys. Eng. Sci., 362 (2004), pp. 493-524.

[9] J. S. Hesthaven and T. Warburton, Discontinuous Galerkin methods for the time-domain Maxwell's equations: An introduction, ACES Newsletter, 19 (2004), pp. 10-29.

[10] I. Mysovskih, Proof of the minimality of the number of nodes in the cubature formula for a hypersphere, USSR Comput. Math. and Math. Phys., 6 (1966), pp15-27.

[11] A. Stroud, Remarks on the disposition of points in numerical integration formulas, Math. Comput., 11 (1957), pp. 257-261.

[12] A. Stroud, Approximate Calculation of Multiple Integrals, Prentice-Hall, Englewood Cliffs, NJ, (1971).

[13] D. Xiu and J. S. Hesthaven, High order collocation methods for differential equations with random inputs, SIAM J. Sci. Comput., 27 (2005), pp. 1118-1139 